EPJ Web of Conferences 116, 03002 (2016)

DOI: $10.1051 /$ epjconf/201611603002

(C) Owned by the authors, published by EDP Sciences, 2016

\title{
Fiber optic hydrophones for acoustic neutrino detection
}

\author{
E.J. Buis ${ }^{1, a}$, E.J.J. Doppenberg ${ }^{1}$, R. Lahmann ${ }^{2}$, P.M. Toet ${ }^{1}$, and J. de Vreugd ${ }^{1}$ \\ ${ }^{1}$ TNO Technical Sciences, PO Box 155, 2600 AD Delft, The Netherlands \\ ${ }^{2}$ Erlangen Centre for Astroparticle Physics, University of Erlangen, Erwin-Rommel-Str. 1, \\ 91058 Erlangen, Germany
}

\begin{abstract}
Cosmic neutrinos with ultra high energies can be detected acoustically using hydrophones. The detection of these neutrinos may provide crucial information about then GZK mechanism. The flux of these neutrinos, however, is expected to be low, so that a detection volume is required more than a order of magnitude larger than what has presently been realized. With a large detection volume and a large number of hydrophones, there is a need for technology that is cheap and easy to deploy. Fiber optics provide a natural way for distributed sensing. In addition, a sensor has been designed and manufactured that can be produced cost-effectively on an industrial scale. Sensitivity measurements show that the sensor is able to reach the required sea-state zero level. For a proper interpretation of the expected bipolar signals, filtering techniques should be applied to remove the effects of the unwanted resonance peaks.
\end{abstract}

\section{Introduction}

The detection of ultra high energy neutrinos, with an energy above $10^{18} \mathrm{eV}$, may provide crucial information about the GZK mechanism [1,2], but could also point to violent and distant astrophysical objects. The expected flux of these cosmic neutrinos, however, is expected to be low, so that large scale neutrino telescopes are needed for this emerging field of astroparticle physics.

Acoustic signals in water induced by energetic neutrino interactions [3, 4] may provide a handle to detect these cosmic particles using hydrophones. Such an acoustics detection method profits from the fact that sound can travel for some kilometers with only small attenuation in the relevant frequency range. A large scale neutrino telescope can therefore be established using hydrophone arrays in the deep sea.

In this paper we discuss the developments on a hydrophone sensor in view of its application in a very large network. We explore the feasibility of manufacturing hydrophone sensors, according to the requirements on the sensitivity and dynamic range, on an industrial scale.

\footnotetext{
${ }^{a}$ e-mail: ernst-jan.buis@tno.nl
}

This is an Open Access article distributed under the terms of the Creative Commons Attribution License 4.0, which permits unrestricted use, distribution, and reproduction in any medium, provided the original work is properly cited. 

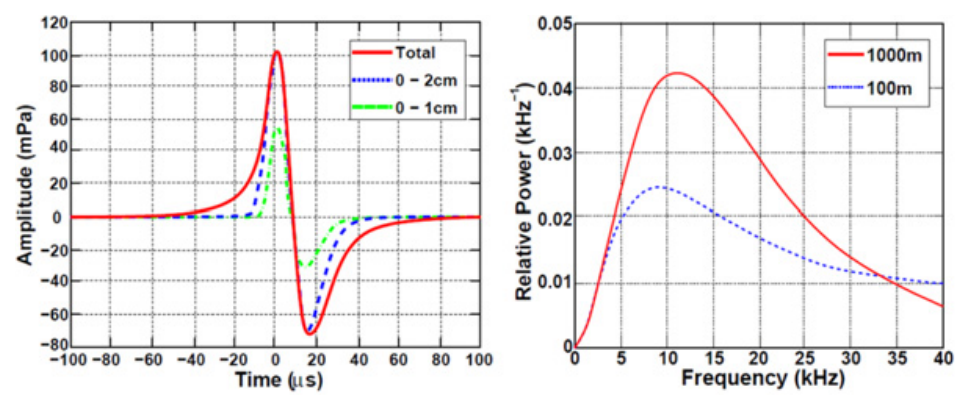

Figure 1. Left: Expected signal from a $1 \mathrm{EeV}$ neutrino shower at a distance of $1 \mathrm{~km}$ (with relative contributions from the shower axis). Right panel: (relative) power spectral density function of an acoustics signal sensed at a distance of $100 \mathrm{~m}$ and $1 \mathrm{~km}$. Adapted from [6].

\section{Acoustic neutrino detection}

The acoustic signals of particle interactions in water have been studied through extensive and detailed simulations, which have been validated by experimental measurements using particle and laser beams $[5,6]$. The acoustic signal expected expected for a $1 \mathrm{EeV}$ neutrino interaction at a $1 \mathrm{~km}$ distance from the sensor is shown in Fig. 1. The shape of the signal is the well-known bipolar pulse, which is characteristic for a neutrino event. In the right panel of the same figure the power spectral density is plotted, which shows that the spectrum peaks around $10 \mathrm{kHz}$, but extends up to $40 \mathrm{kHz}$ and higher.

As the acoustic source, i.e. the particle shower, can be approximated by a thin line source, the acoustic pattern that is formed in the water has a shape of a pancake: the pattern is, according the length of the particle shower, about 10-20 m thick, but may extend to several km's in radial direction. The radial exention of the accoustic pressure field is due large absorption length of sound, which is about $1 \mathrm{~km}$ at $25 \mathrm{kHz}$, depending on salinity and temperature [7]. The distinct acoustic pattern will prove crucial in event reconstruction to discriminate a neutrino event from background as well as to obtain the direction of the neutrino.

\section{Fiber based neutrino telescope}

It is clear from the various discussions in literature that a(n acoustic) neutrino telescope suitable for the study of ultra-high energy neutrinos is required to have a detection volume of at least $100 \mathrm{~km}^{3}$ or more [8]. In addition, such a volume should be equipped with at least 1000 hydrophones for a proper event reconstruction. Such a detection volume demands a truly Very Large Volume neutrino Telescope $(\mathrm{VLV} v \mathrm{~T})$.

\subsection{Detection concept and technology}

It has been advocated that fiber optic hydrophone technology is a promising means to establish a sensitive, cost-effective and large scale sensor network [9-11], that could provide the basis of a future neutrino telescope. This technology is based on fiber lasers and hydrophone sensors that are integrated on a single optical fiber as shown in Fig. 2. The sensors transform the acoustic pressure into strain in the fiber. At the position of each sensor a fiber laser is inserted in the optical fiber, which emits light of a well-known wavelength. The strain causes a wavelength shift of the laser light and is sensed using an interrogator. The interrogator houses an interferometer to determine the wavelength shift, but also a pump laser to pump all the fiber lasers in the string. The readout scheme of the interrogator is 


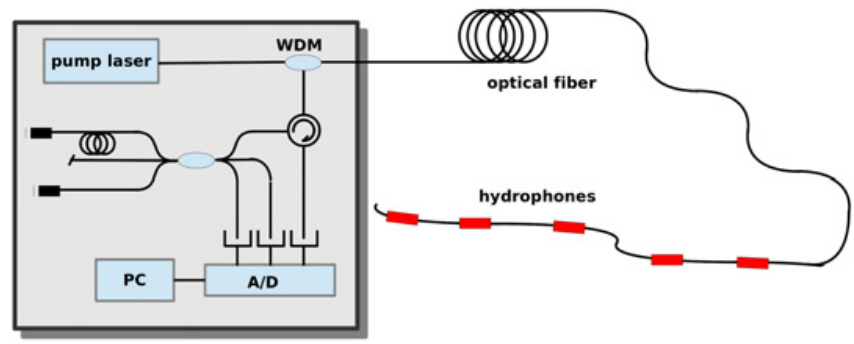

Figure 2. Concept of the optical fiber sensor network: An optical fiber with several fiber lasers and sensors are read out using an interrogator. Once deployed in the deep-sea, several of these strings make up a neutrino telescopes.
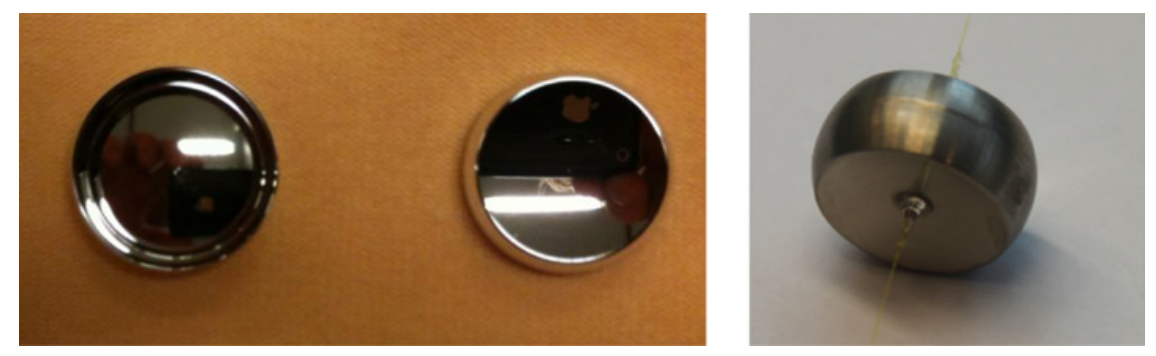

Figure 3. Left photograph: two halves of the sensor. In the photograph on the right the sensors is assembled and glued to an optical fiber.

plotted in Fig. 2. Note that the distance between the sensors and the interrogator can be large, so that the interrogator can be located on shore or on any other convenient location.

\subsection{Sensor development for a VLVvT}

An attempt was made to develop a sensor that is cost effective and can be mass produced. The design consisted of two halves made out of aluminum as shown in Fig. 3. The aluminum parts were constructed using a turning machine, but could in future be produced through the method of die cutting. Such a construction method allows for a mass scale production of the sensor.

During assembly, the two halves were glued and an optical fiber was guided through a hole in the center of the sensor as shown in the right panel of Fig. 3. At the time of the measurements, no fiber lasers of the size that match the dimensions of the sensor were available, so that simple and less sensitive fiber bragg gratings (FBG) were used instead. As a concequence, no pulses could be recorded at the $\mathrm{mPa}$ level (Fig. 1), but a characterization of the sensor could be done nevertheless on the overall response as a function of the acoustic frequency to study the mechanical resonances.

\section{Results}

The sensor as described in the previous section has been tested in an anechoic basin at TNO. A B\&K8103 type hydrophone from Bruël and Kjaer was taken as a reference hydrophone that has a flat response at the relevant acoustic frequencies. The response of the hydrophone was determined as a function of the acoustic frequency and is shown in Fig. 4 (left panel). The graph shows two large resonance peaks that are due to the eigen frequencies of the aluminum housing as well as the fiber that is attached to it. A filter has been applied to remove these peaks of which the result is shown in the right 

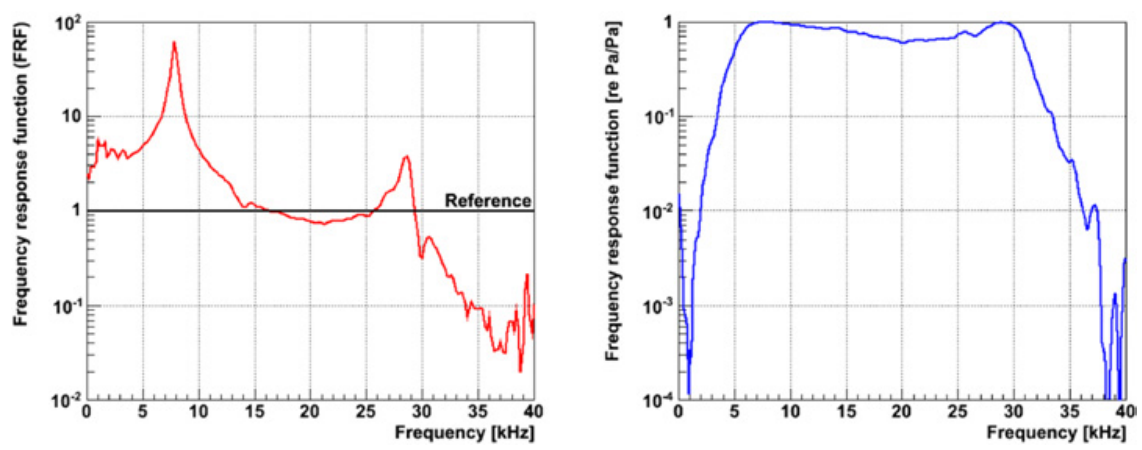

Figure 4. Measured response of a sensor (shown in Fig. 3 prior (left) and after filtering (right).

panel of the figure. Here, the Box-Jenkins method was applied (see e.g. [12]). It can be seen that the filtering is quite effective and that the peaks disappear and the response is relatively flat in the region of interest.

\section{Conclusions}

Within the field of astroparticle physics, the study of ultra-high energy neutrinos may come within reach when neutrino telescopes can be constructed using large scale fiber optic networks. Such a network, when equiped with a large number of hydrophones, would allow to detect the cosmic neutrinos through the acoustic signals that the may induce in the deep sea.

We have designed, realized and characterized a new hydrophone sensor based on fiber lasers that can be produced cost-effectively on a mass-scale. It was found that the sensor exhibits two resonance peaks that are related to the aluminum housing of the sensor as well as the fiber that is attached to it. Filtering has been applied to obtain a flat response of the sensor, which is required for a proper interpretation of the data.

\section{References}

[1] K. Greisen, Physical Review Letters 16, 748 (1966)

[2] G.T. Zatsepin, V.A. Kuz'min, ZhETF Pisma Redaktsiiu 4, 114 (1966)

[3] G.A. Askaryan, Zemlia i Vselennaia 1, 13 (1979)

[4] J.G. Learned, Phys. Rev. D 19, 3293 (1979)

[5] L. Sulak, et al., Nuclear Instruments and Methods 161, 203 (1979)

[6] S. Bevan, et al. (Acorne Collaboration), Astroparticle Physics 28, 366 (2007)

[7] M.A. Ainslie, J.G. McColm, Acoustical Society of America Journal 103, 1671 (1998)

[8] E. Waxman, Science 315, 63 (2007)

[9] P.E. Bagnoli, et al., Nuclear Instruments and Methods in Physics Research A 567, 515 (2006)

[10] E. Marconi, et al., Nuclear Instruments and Methods in Physics Research A 572, 490 (2007)

[11] E.J. Buis, et al., Journal of Instrumentation 9(03), C03051 (2014)

[12] L. Ljung, ed., System Identification (2Nd Ed.): Theory for the User (Prentice Hall PTR, Upper Saddle River, NJ, USA, 1999), ISBN 0-13-656695-2 